Final draft of Suthers, D. D., Dwyer, N., Medina, R., \& Vatrapu, R. (2007). A framework for eclectic analysis of collaborative interaction. In C. Chinn, G. Erkens \& S. Puntambekar (Eds.), The Computer Supported Collaborative Learning (CSCL) Conference 2007 (pp. 694-703). New Brunswick: International Society of the Learning Sciences.

\title{
A Framework for Eclectic Analysis of Collaborative Interaction
}

\author{
Daniel Suthers, Nathan Dwyer, Richard Medina, Ravi Vatrapu \\ Laboratory for Interactive Learning Technologies \\ Dept. of Information and Computer Sciences, University of Hawai i at Manoa \\ 1680 East West Road, POST 309, Honolulu, HI 96822, USA \\ collaborative-representations@hawaii.edu
}

\begin{abstract}
The interactional structure of learning practices is a central focus of study for CSCL, although challenges remain in developing and pursuing a systematic research agenda in the field. Different analytic approaches produce complementary insights, but comparison is hampered by incompatible representations of the object of study. Sequential interaction analysis is promising but must be scaled to distributed and asynchronously mediated settings. Building on recent analytic work within our laboratory, we propose a framework for analysis that is founded on the concepts of media coordinations and uptake, and utilizes an abstract transcript representation, the dependency graph, that is suitable for use by multiple analytical traditions and supports examination of sequential structure at larger scales.
\end{abstract}

\section{Introduction}

Learning in collaboration with others is the foundation of Computer Supported Collaborative Learning (CSCL). An overview of the historical development of the field (Stahl, Koschmann, \& Suthers, 2006) reveals the presence of several research traditions, including an analytic tradition that began with a conception of collaboration as a "continued attempt to construct and maintain a shared conception of a problem" (Roschelle \& Teasley, 1995). More recently, there have been calls to focus work in the field on the study of "the practices of meaning-making in the context of joint activity" (Koschmann, 2002) or "intersubjective meaning-making" (Suthers, 2006b), from which "group cognition" (Stahl, 2006) emerges. The common emphasis is on the interactional structure of collaborative learning. Diverse lines of work exist in CSCL: we study interaction in different media, examine phenomena ranging from micro-episodes in small groups to large communities over periods of weeks to months, and analyze data using various "qualitative" and "quantitative" analytic approaches. In order to enable the cross-pollination of these different lines of work, there is a need for shared definitions and a common formalism. The work in our own laboratory spans some of this diversity, including study of co-present and distributed interaction with various synchronous and asynchronous media, and applying experimental and ethnographic methodologies at scales including pairs, small groups and online communities. Because of this diversity in our own work, we have encountered the need for greater theoretical and methodological dialogue; a need that also exists in the field of CSCL as a whole. We are committed to studying collaborative learning as a mediated interactional accomplishment, but wish to do so in settings beyond micro-episodes of synchronous interaction, and to apply a mixture of methods for hypothesis generation and testing. In this paper, we report on a framework for eclectic analysis of collaborative interaction that we have developed for our own work, in hopes that adoption by others may increase dialogue within the field as well. The framework is based on the concept of uptake and a few associated theoretical commitments that are necessary to define interaction as a common object of study. The primary feature of this framework is an abstract transcript notation-the dependency graph-that offers a common representational basis for diverse analytic methods applied to various media and interactional situations. The remainder of this paper documents the motivations, theoretical foundation, and practical aspects of the framework that has resulted, with selected examples.

\section{Motivations}

The approach is based on several years of our own analytic work, initiated to expose the practices of mediated collaborative learning in data from our prior experimental studies. In an analysis undertaken in order to understand how knowledge building was accomplished via synchronous chat and evidence mapping tools, we used the concept of uptake to track interaction distributed across these tools (Suthers, 2006a). Subsequently, we began analyzing asynchronous interaction involving threaded discussion and evidence mapping tools (Suthers, Dwyer, Vatrapu, \& Medina, 2007). The uptake analytic framework was further developed to handle the asynchronicity and multiple workspaces of these data. Below we summarize the view of learning underlying our current work, assess prevalent analysis methodologies in relation to our needs, and then discuss additional requirements for eclectic methodologies. 
Final draft of Suthers, D. D., Dwyer, N., Medina, R., \& Vatrapu, R. (2007). A framework for eclectic analysis of collaborative interaction. In C. Chinn, G. Erkens \& S. Puntambekar (Eds.), The Computer Supported Collaborative Learning (CSCL) Conference 2007 (pp. $694-703)$. New Brunswick: International Society of the Learning Sciences.

\section{Learning as an Interactive Process}

Although we believe that the framework we offer in this paper can support analyses under a variety of views of learning, the framework is motivated by our own views of how learning takes place in social settings. We conceive of learning as an interactional process of change. This conception of learning as interactional is compatible with theories of learning that identify individuals (Beck, 1997; Chi, Bassok, Lewis, Reimann, \& Glaser, 1989), socially embedded individuals (Doise \& Mugny, 1984; Vygotsky, 1978), social systems (Engestrom, 2001), or communities (Wenger, 1998) as the locus of change. Learning need not be deliberately sought: it is a result of participants' attempts to make sense of a situation. Meaning-making, as we call it in this paper, takes place at multiple levels: solving a problem, maintaining interpersonal relationships, and/or affirming identity in a community (Bronckart, 1995). To study learning in social settings we must necessarily study individual trajectories of meaningmaking and how they intertwine in practices of intersubjective meaning-making (Suthers, 2006b). In such settings, the meaning of a given contribution is best understood as a function of its relationships to prior interactions, and indexically with respect to the physical and social context (Koschmann, Zemel, \& Stahl, 2004). Meaning-making is mediated by the physical and social environment in diverse ways (Wenger, 1998; Wertsch, 1998). As designers of media for online learning, this mediation gives us an avenue for influencing meaning-making and learning through the social affordances of the tools that we design (Suthers, 2006b).

\section{Statistical Aggregation}

Many approaches to the study of learning follow a quantitative paradigm in which contributions (or elements of contributions) are annotated according to a well-specified coding scheme (e.g., De Wever, Schellens, Valcke, \& Van Keer, 2006; Rourke, Anderson, Garrison, \& Archer, 2000). Statistical methods are then used to characterize aggregate behaviors that may then be compared across experimental conditions. This approach has three significant strengths. First, a coding scheme is a concrete classification of behaviors that supports mathematical methods for estimating consistency (reliability) between multiple analysts. Second, the approach has well defined statistical methods for comparing results from multiple sources of data such as experimental conditions and replications of studies. Third, this approach can scale up analysis by quantifying data across large groups. The trade-off is that "coding and counting" obscures the sequential structure and situated methods of the interaction. "Coding" assigns the meaning of an act as an isolated unit, and therefore either does not take the indexicality of this meaning into account or fails to record the evidence on which the analyst relied in making a judgment. "Counting" or statistical aggregation loses the sequential methods by which media affordances are used in particular learning accomplishments, making it more difficult to identify important design elements at the same temporal and spatial grain as the actual interaction itself.

\section{Sequential Analysis}

A contrasting approach finds the significance of each act in the context of the unfolding interaction. This approach includes Conversation Analysis (Goodwin \& Heritage, 1990; Sacks, Schegloff, \& Jefferson, 1974) and Interaction Analysis (Jordan \& Henderson, 1995). Typically, these methods repeatedly examine the micro-structure of short interaction segments to uncover the methods by which participants make their actions accountable to each other (Garfinkel, 1967). This approach is a complement to statistical aggregation and has the opposite strengths and weaknesses. These methods document the actual practices of learning by attending to the sequential structure of the interaction, producing detailed descriptions that are deeply situated in the medium of interaction. However, sequential analyses are often time consuming to produce and difficult to generalize to different media or groups. A micro-analysis can capture sequential properties because analysis is focused on short interactions that an analyst can view and review, but progressively larger structures escape its grasp. The family of methods loosely classified as "exploratory sequential data analysis" (Sanderson \& Fisher, 1994) address some of these concerns with computational support for statistical and grammatical analysis (Olson, Herbsleb, \& Rueter, 1994).

\section{Additional Requirements}

The different environments and media under examination have spawned multiple environment- and medium-specific analytic notations. For example, ethnography relies to a large extent on freeform notes taken by observers. Studies of conversation have used simple transcripts of utterances (Roschelle, 1992) and more detailed transcripts using Jeffersonian notation (Sacks et al., 1974). Video has become the standard recording medium for studies of practice (Jordan \& Henderson, 1995; Koschmann et al., 2004). Video analysis tools (e.g., Pea, 2006; Woods, 2006) provide support for exploring and annotating video records, but the annotations are tied to this medium. Online interaction simplifies the creation of transcriptions: software tools can record a detailed and 
Final draft of Suthers, D. D., Dwyer, N., Medina, R., \& Vatrapu, R. (2007). A framework for eclectic analysis of collaborative interaction. In C. Chinn, G. Erkens \& S. Puntambekar (Eds.), The Computer Supported Collaborative Learning (CSCL) Conference 2007 (pp. $694-703)$. New Brunswick: International Society of the Learning Sciences.

comprehensive log of an interaction. However, online media introduce asynchronicity and hide the production of contributions (Clark \& Brennan, 1991), introducing different demands on analytic notations. Analysis of the simultaneous use of many communication media and channels has relied on ad hoc, eclectic representations (see, for example, Hmelo-Silver, 2003; Suthers, 2006a). Because interaction relies on many different semiotic resources, analysis of interactional processes must be sensitive to the social affordances of the specific medium being analyzed, yet also be applicable across multiple media in order to facilitate dialog between researchers. This introduces a pair of related challenges to the creation of a generalizable method: it must be media agnostic but simultaneously media aware. A workable method needs to be independent of the form of the data under analysis. At the same time, the method needs to maintain a record of how people make use of the specific affordances of media. This is required to allow analysis to speak to design and empirically drive the creation of new, more effective media.

Much of the foundational work in sequential analysis of interaction has focused on face-to-face interaction. Production blocking and the ephemerality of spoken interactions constrain communication in such a manner that turns (Sacks et al., 1974) and adjacency pairs (Schegloff \& Sacks, 1973) are appropriate units of analysis for face-toface data. These units of analysis are not as appropriate for CMC since most online media support simultaneous production and persistence of contributions. Contributions may become available to other participants in unpredictable orders, may not be immediately available, and may address earlier contributions at any time (Garcia \& Jacobs, 1999; Herring, 1999). Because conceptual coherence can be decoupled from temporal or spatial adjacency, we cannot restrict analysis to the relationships between adjacent events. Nor is it appropriate to treat CMC as a degenerate form of face-to-face interaction (e.g., by seeking an analog to adjacency pairs) since people use attributes of new media to create new forms of interaction (Dwyer \& Suthers, 2006; Herring, 1999).

Based on considerations discussed in this section, we sought an analytic approach that (1) maintains the sequential and situational context of activity so that an account of the interactional construction of meaning is possible, (2) does not assume that the medium of interaction has any particular interactional properties (e.g., synchronicity, availability of contributions, or persistence), but (3) records these properties where they exist. Additionally, it should (4) be sufficiently formalized to enable computational support for analysis, including sequential and statistical analysis, and (5) capture aspects of interaction that are critical to learning. The analytic framework we developed draws on other interaction analysis methods, but it uses generalized concepts of interaction elements and structures that are independent of any particular medium. The remainder of the paper describes the theoretical foundations for our analytic representation, and how it is constructed and used.

\section{Theoretical Foundations}

We need a unit of interaction that abstracts from media-specific concepts such as adjacency, is applicable to the wide variety of temporal, spatial and notational properties of media, and is capable of tracing the entwinement of individual and intersubjective trajectories of meaning-making. Since collaborative learning is only possible when something is shared and transformed between participants, we built this unit of analysis on the concept of uptake (Suthers, 2006a). Uptake is how we describe the act of a participant taking reifications of prior or ongoing participation (e.g., expressions of information, attitudes and attentional orientation; whether ephemeral or persistent) as having certain relevance for further participation. Uptake is a transitive act, in that it always is oriented towards the taken-up as its object, which is foregrounded by the act as being relevant. Uptake is interpretative: some particular aspect of the object is brought forth and given (further) meaning. The "thematic connections" of Resnick, Salmon, Zeitz, Wathen, \& Holowchak (1993) are an example of uptake, although our conception allows for nonlinguistic forms of expression, and for other kinds of interpretative acts in addition to argumentative ones. A participant can take up one's own prior reifications as well as those of others: by identifying both, analysts can characterize visible trajectories of intrasubjective and intersubjective meaning-making. Uptake is a form of participation: the act must be visible within a given realm of participation to be uptake in that realm. An individual working through ideas via mental processes and external notations has access to his or her uptake across as well as within these media, but in the social realm only visible acts can foreground and interpret prior reifications.

Our framework for uptake analysis tries to be useful to multiple theoretical and analytic paradigms, but is based on two theoretical assumptions about the nature of artifact-mediated collaborative interaction.

- Coordination: Efforts to coordinate between the personal and social realms are enacted through media (including expressions and perceptions).

- Ongoing sequential structure: The sequential structure of these coordinations at successively overlapping and expansive granularities is significant in understanding how meaning-making is accomplished. 
Final draft of Suthers, D. D., Dwyer, N., Medina, R., \& Vatrapu, R. (2007). A framework for eclectic analysis of collaborative interaction. In C. Chinn, G. Erkens \& S. Puntambekar (Eds.), The Computer Supported Collaborative Learning (CSCL) Conference 2007 (pp. $694-703)$. New Brunswick: International Society of the Learning Sciences.

All interaction is mediated by physical and cultural tools (Wertsch, 1998), whether in ephemeral media such as thought, speech and gesture, or persistent media such as writing, diagrams, or electronic representations. Distributed cognition (Hutchins, 1995) describes how information is transformed as it propagates via coordinations of representations through a distributed socio-technical system. According to Hutchins, the coordinated representations include individuals' internal conceptions in addition to external, perceptible representations. We draw on the idea of coordination, noting that coordination between personal and social realms can be accepted regardless of whether one accepts the existence of cognitive representations. A typical distributed cognition analysis starts by identifying a system's function (e.g., steering a ship) and involves tracing the propagation of information through the system and identifying transformations that take place at points of coordination between the participants and external representations. In settings fundamentally concerned with the creation of new knowledge, this focus on the enactment of functional relationships implies too static an interaction structure, and indeed takes as a starting point that which analysis seeks to uncover. An analysis based on uptake, in contrast, starts with the identification of acts of coordination and the dependencies between them, and seeks to recognize what is accomplished through the interaction. In doing so, we draw on the ethnomethodological idea that the meanings of actions are indexical (deeply tied to the time and place of their enactment), and the consequence that the sequential structure of activity is of fundamental importance (Garfinkel, 1967; Koschmann et al., 2004).

Motivated by the need for a common transcript representation that exposes interactional structures in diverse forms of mediated interaction, and for a formal structure that is amenable to computation, we developed the dependency graph. A schema for the basic analytical elements is shown in Figure 1. Any empirical analysis must be built upon observable events. We assume that an analyst is interested in deliberate acts, not just any physical event. Therefore the analyst will examine the ongoing stream of events and identify those that appear to be coordinations between the

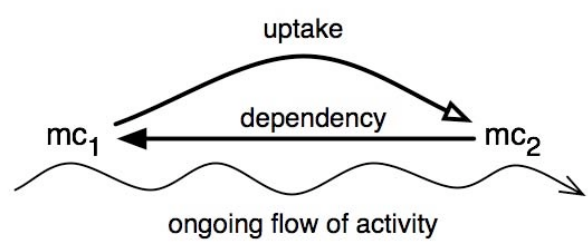

Figure 1. Schema for a dependency personal and public realms. These media coordinations are exemplified by $\mathrm{mc}_{1}$ and $\mathrm{mc}_{2}$ in Figure 1 . The existence of conceptions is implied by media coordinations, but we need not (yet) identify these conceptions (see Suthers, Dwyer et al., 2007 for further discussion of implied conceptions). The analyst need only make a commitment that certain coordinations are of interest.

If a media coordination $\mathrm{mc}_{2}$ is to be understood as taking up the contribution of a prior coordination $\mathrm{mc}_{1}$, then there must be some observable relationship between the media coordinations. Therefore, we further ground the uptake analysis in empirical evidence by identifying dependencies between media coordinations that suggest that there is uptake. Dependencies can be found in media-level, representational, and semantic relationships between media coordinations: these will be discussed below. The dependency graph representation takes the form of a directed acyclic graph consisting of media coordinations and the dependencies between them (see Suthers, 2006a for a formal definition) on which we may layer analytic interpretations. Dependencies provide evidence that uptake may exist, but not all dependencies as defined at the media level need be uptake. The distinction between dependencies and uptake is made because dependencies reflect the myriad of ways in which human action is deeply embedded in and sensitive to the environment and immediate history of interaction, while only some of these relationships enter into the realm of meaning in which participants are demonstrably oriented towards reifications as having relevance for ongoing participation. Once these relationships have been identified, the graph defined by reversing the arcs may be properly called an uptake graph, as in (Suthers, 2006a).

Although we have described uptake as something that participants do, uptake is more accurately understood as an etic abstraction that we as analysts use to identify interactionally significant relationships between acts. From an emic perspective, participants don't engage in the abstract act of uptake; they engage in specific acts that they affirm (through subsequent activity) as taking up prior acts in the accomplishment of recognizable activity (Garfinkel, 1967). The analyst's identification of uptake is a bridge between empirical dependencies and further analysis. Uptake analysis is a proto-analytic method that must be completed by further analysis motivated by a given research program. The dependency graph provides resources for this further analysis by offering potential instances of uptake and grounding analysis in empirical media coordinations. This representation can support multiple methods of analysis, is amenable to computational support and visualization, and is meant as a boundary object for discussion and collaboration across different analytical traditions. 
Final draft of Suthers, D. D., Dwyer, N., Medina, R., \& Vatrapu, R. (2007). A framework for eclectic analysis of collaborative interaction. In C. Chinn, G. Erkens \& S. Puntambekar (Eds.), The Computer Supported Collaborative Learning (CSCL) Conference 2007 (pp. $694-703$ ). New Brunswick: International Society of the Learning Sciences.

\section{Uptake Analysis}

This section describes the practical tasks involved in producing and interpreting a dependency graph, accompanied by a discussion of related issues and concrete examples from our analysis work. In practice, the process may iterate between identification of media coordinations, dependencies, and uptake; and may be driven by specific analytic goals or may be more exploratory in nature.

\section{Identifying Media Coordinations}

A dependency graph is built on observed media coordinations for which conceptual or interactional significance is claimed. Media coordinations are a more general form of elements from other analytical methods. Content analysis methods that work with text highlight and code elements in the text record. Conversation analysis and video-based micro-analysis identify points of interest in the media recording or transcript, and the media or transcript may be similarly coded or annotated. The analyst's identifications of media coordinations fulfill the same function as these annotations. Media coordinations are represented as vertices in the dependency graph. We call these vertices fixed points since they constitute the points of departure for analysis. Fixed points are anchored in media coordinations that can vary in granularity from a single instant to a period of time. The fixed point's anchor should be specific enough to allow the analyst to return to the media action as accounted in the data record. As in most interaction analysis methods, the source data is always the final authority.

Some media coordinations are easy to identify. When analyzing spoken conversation or CMC, utterances and messages are obvious candidates for media coordinations. The creation of an object in a shared workspace is similarly easy to identify as a media coordination. We use the general term expressions to refer to media coordinations of this nature. Other media coordinations are less obvious. For example, if two items are placed near each other in a workspace this may be an expression of relatedness (Dwyer \& Suthers, 2006). This illustrates the more general issue of not confusing the representational vocabulary of a medium with the actions supported by the medium. For example, a medium that supports spatial positioning may be used to create groups even if no explicit grouping tool is provided.

Perceptions (e.g., hearing or reading another's expression) are another form of coordination between representation and conception. Explicit identification of perception is absent from many other analysis methods, which implicitly assume that each participant perceives every contribution, and does so at the time that it is produced or displayed. With asynchronous data this assumption is clearly untenable. The applicability of this assumption to synchronous interaction can also be questioned. Therefore our abstract transcript representation allows for explicit specification of evidence for perceptions as another form of media coordination. It is difficult to identify the conception that results from a perception, but it is sufficient to assume that some conception results and mark the perception event as a media coordination. Researchers interested only in public behavior need not go further than to use the perceptual media coordination to narrow the temporal scope of uptake of the perceived contribution. Researchers interested in psychological (e.g., cognitive) claims about individual learning may subsequently attempt to infer the conception based on other evidence, including dependency relations. In either case, the observed evidence for perceptual coordinations has been made explicit.

A fixed point is incomplete without a description of the evidence on which the analyst based its identification. The practice of making evidence explicit addresses several issues. It limits the degree to which analysts can make assumptions about media coordinations. For example, maintaining the distinction between expression and perception has forced us to question our assumptions about which contributions are available to others. Specifying the evidence distinguishes the descriptive "what" of the interaction from the explanatory "why" of the analyst's interpretation, making clear the specific details that were seen as significant. This helps multiple analysts collaboratively review their observations and interpretations and facilitates trans-disciplinary discussions.

\section{Identifying Dependencies}

The second task in constructing a dependency graph is to identify and document the dependencies between media coordinations. A dependency represents a grounded assertion that the media coordination identified by one fixed point enables the media coordination identified by another fixed point. Dependencies map out the sequential unfolding of the interaction. They are defined in terms of a set of participating media coordinations and grounded evidence for their interdependencies. 
Final draft of Suthers, D. D., Dwyer, N., Medina, R., \& Vatrapu, R. (2007). A framework for eclectic analysis of collaborative interaction. In C. Chinn, G. Erkens \& S. Puntambekar (Eds.), The Computer Supported Collaborative Learning (CSCL) Conference 2007 (pp. 694-703). New Brunswick: International Society of the Learning Sciences.

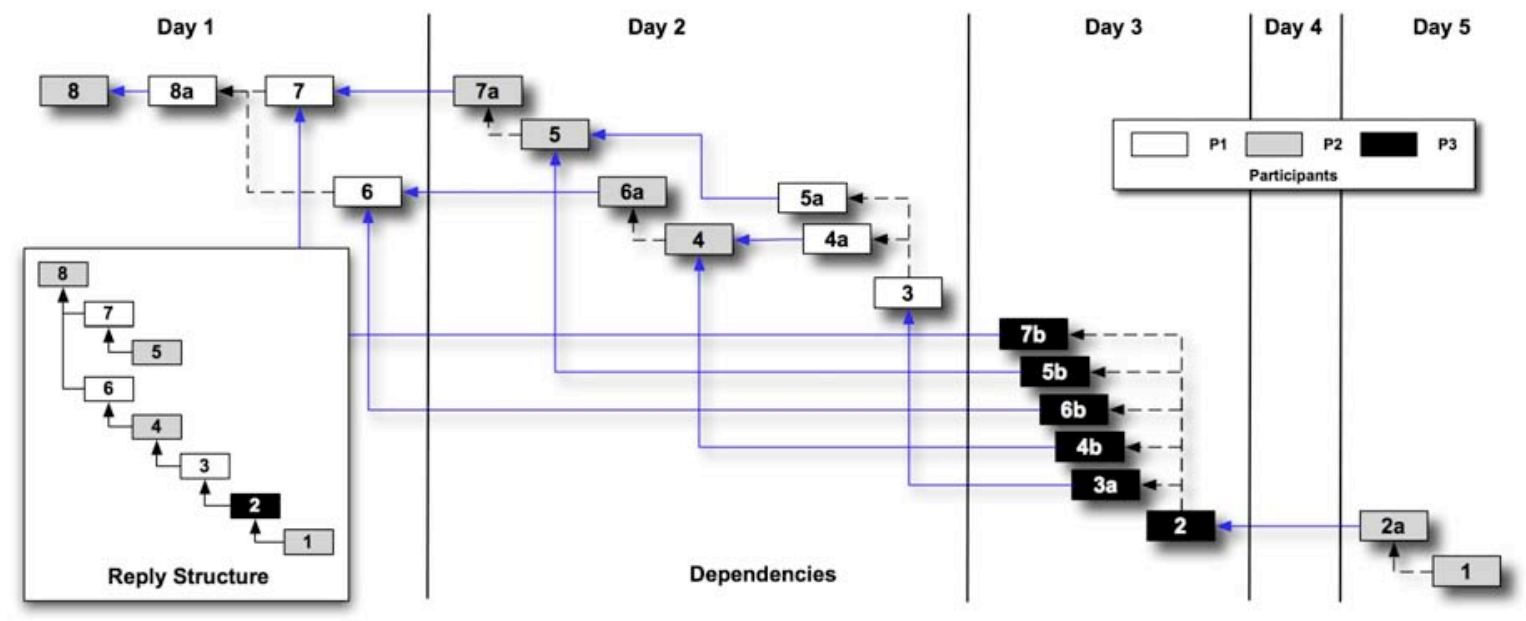

Figure 2. Comparison of threaded discussion reply structure (inset) and dependency graph (main graph) from an online discussion. Fixed points without letters are evidenced by message postings and with letters are evidenced by message reads. Dashed lines represent dependencies for intrasubjective uptake.

Two or more media coordinations can participate in a dependency relationship. Dependencies are directional and point backwards in time. A dependency expresses how a single media coordination depends on one or more prior media coordinations. If multiple coordinations are dependent on a single coordination, then multiple dependencies are specified. If $\mathrm{mc}_{2}$ depends on $\mathrm{mc}_{1}$ then we are claiming that $\mathrm{mc}_{1}$ enabled $\mathrm{mc}_{2}$, but there is no assertion that $\mathrm{mc}_{1}$ caused $\mathrm{mc}_{2}$. In our work we have frequently had to work with the ambiguity of "potential dependencies". Dependencies are a generalization of relationship types from other sequential data analysis methods, such as "adjacency pairs," "reply," "thematic connections," etc., and are candidate uptakes. Specifying the evidence for the dependencies serves the same purpose as for the fixed points. In particular, explicit examination of the evidence makes it easier to distinguish the assertion of the dependency from its interpretation. In contrast, in many coding methods the analyst simply asserts an interpretation, e.g., that a contribution is an "elaboration" on or "objection" to another, and the validity of this interpretation is established through computations of inter-rater reliability that do not make the evidence explicit. With dependencies, the evidence must support the assertion that one or more media coordinations played a role in enabling another media coordination. Some types of evidence are more easily identified than others. We have used three types of evidence for dependencies in our work. Starting with the most concrete they are media dependencies, representational association, and semantic relatedness. These are discussed below along with examples.

The most concrete evidence is in the form of media dependencies - one action on the representation could not have taken place in the absence of a previous action. A reply in a threaded discussion depends on the prior existence of the message being replied to, and modifying an element of a shared workspace depends on the previous act of creating the element. However, care must be taken not to fall into the trap of conflating the representational vocabulary with the steps in the interaction. Consider a reply in a threaded discussion. The reply message is dependent on the message being replied to, but in terms of dependencies between coordinations it is more accurate to say that the creation of the reply message is dependent on the author's perception of the message being replied to. Figure 2 (adapted from Suthers, Dwyer et al., 2007) contrasts the reply structure of a short discussion (inset figure) with the dependency structure (including perceptions) from which we inferred uptake (main figure). Nodes with letters such as $8 \mathrm{a}, 7 \mathrm{~b}$, etc. represent media coordinations evidenced by message read events. When these perceptionrelated media coordinations are included, a much different pattern emerges. In particular, participant 3's posting (fixed point 2) is not only related to the single message being replied to, but is the result of a series of reads that encompasses two subthreads of the discussion.

The second type of dependency evidence is representational association. The use of similar representational attributes is often used to indicate relatedness (Dwyer \& Suthers, 2006). The representations can have similar visual attributes (e.g., color or type face) or they can be grouped together or aligned spatially. Temporal proximity can also indicate relatedness-expressions that follow each other closely are often part of the same exchange. Each of these indications of relatedness can imply a dependency. In Figure 2, temporal proximity is part 
Final draft of Suthers, D. D., Dwyer, N., Medina, R., \& Vatrapu, R. (2007). A framework for eclectic analysis of collaborative interaction. In C. Chinn, G. Erkens \& S. Puntambekar (Eds.), The Computer Supported Collaborative Learning (CSCL) Conference 2007 (pp. 694-703). New Brunswick: International Society of the Learning Sciences.

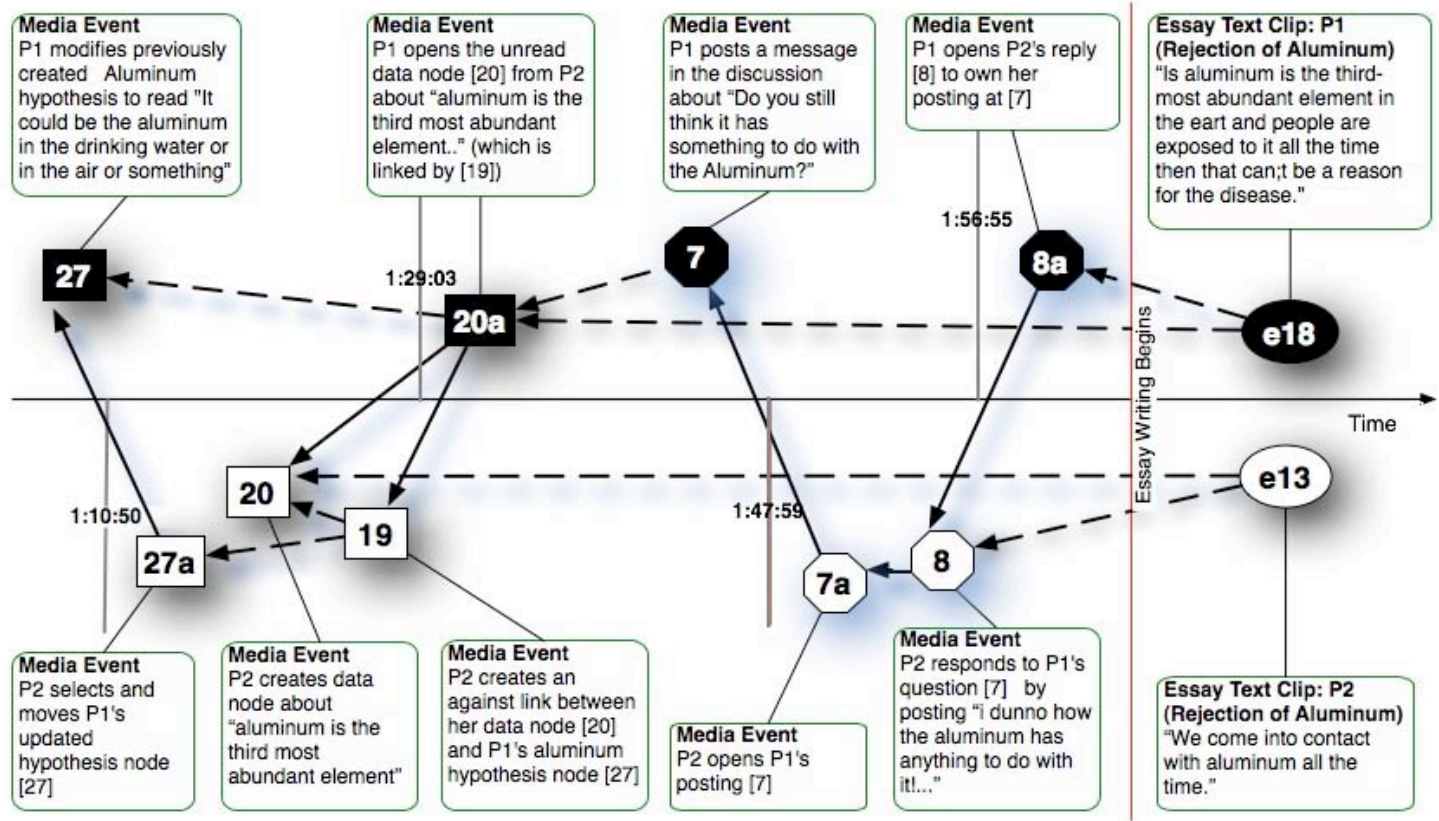

Figure 3. Dependency graph of a dyad collaborating asynchronously with multiple media. Participant 1's coordinations are above and Participant 2's coordinations are below the timeline. Vertical bars represent workspace synchronizations in which the partner's recent work became available. Rectangles, octagons, and ellipses represent coordinations with an evidence map, a threaded discussion, and a word processing tool, respectively. The graph is partial and was constructed by identifying dependencies backwards from the portions of the essays shown.

of our evidence for the dependency of 2 on $7 b, 5 b, 6 b, 4 b$, and 3a. In Figure 3 (an analysis of collaboration through a shared workspace to be discussed below), spatial connectivity is our evidence for the dependency of perception 20a on 19. Representational association can also consist of repeated words and phrases indicating a dependency on the media coordination in which they were introduced. This can sometimes be easy to identify, for example when copy and paste is observed, or a phrase is typed soon after reading it. However, in general it may be more difficult to identify the original source of any content or to determine whether or not its re-use is actually dependent on the prior use.

The final type of evidence is semantic relatedness: the semantic content of a media coordination can be traced to the semantic content of another media coordination. See for example the dependency of 7 on 20a in Figure 3. Semantic dependency can be difficult to identify and is often open to debate. For example, in one case we looked at, one participant added three related nodes to an evidence map. The other participant, after reading them, added a fourth node that seemed to summarize the first three. In general, representational and semantic dependencies are more convincing if convergent evidence exists (e.g., temporal proximity and semantic relatedness).

\section{Documenting other media elements}

A dependency graph is a partial transcription of an interaction. It may be necessary to record additional information to contextualize the interaction. This additional information can annotate or augment the dependency graph formalism. For example, the reply structure of a threaded discussion is an important resource for understanding the participants' view of the medium, and so is included in Figure 2. In (Suthers, Vatrapu, Medina, Joseph, \& Dwyer, 2007), we used an asynchronous protocol. In order to identify which representational elements each participant had available at any point in time, we incorporated indications of workspace updates by which participants received new data from their partner, visualized as vertical bars in Figure 3.

\section{Iteration}

Production of the dependency graph is an iterative process of densification: multiple passes through the data identify additional elements and provide new insights into the interaction. The formalism of the dependency 
Final draft of Suthers, D. D., Dwyer, N., Medina, R., \& Vatrapu, R. (2007). A framework for eclectic analysis of collaborative interaction. In C. Chinn, G. Erkens \& S. Puntambekar (Eds.), The Computer Supported Collaborative Learning (CSCL) Conference 2007 (pp. $694-703)$. New Brunswick: International Society of the Learning Sciences.

graph provides support for this process. New fixed points and dependencies can be continually added to the graph. This has the following ramifications. The graph can grow in complexity to reflect a deepening knowledge of the data, but the graph can never be considered "complete," except with regard to particular representational elements (e.g., it is possible to claim that every discussion posting has been recorded as a fixed point). Therefore, one must be cautious about asserting that a practice or pattern never occurs. The quality of the analysis is proportional to the richness of the data. In our work with threaded discussions for online courses we only have log entries for when a message was created and when a user opened a message. Other media coordinations such as scrolling are not logged. On the other hand, our experimental configuration provides a complete record of every mouse and keyboard event, every action on the shared representation, and a video capture of the computer screen from each client. The richness of the latter data has allowed us to examine interaction at a much finer grain. Nonetheless, the threaded discussion data is sufficient for coarser grained analysis. Finally, repeated iterations may identify new types of representational elements, media coordinatins, and dependencies. Our work has suggested two other constructions: interactionally defined representational elements that do not correspond to any explicit representational notation, and composite media coordinations in which two or more media events seem to share a conception.

\section{Example of Discovery by Uptake Analysis}

Figure 3 presents a dependency graph of data from a study of collaborative argumentation with evidence maps. See (Suthers, Vatrapu et al., 2007) in this volume for details of the study. This analysis was done to understand how two participants used media resources to converge on the conclusion that aluminum is probably not the cause of a disease under consideration. (The relevant information had been distributed across participants in a hidden profile.) See (Suthers, Medina, Vatrapu, \& Dwyer, 2007) in this volume for discussion of whether convergence is achieved by information sharing alone or whether interactional "round trips" are required. Construction of the dependency graph allowed us to discover an interesting interactional pattern that goes beyond simple round trips. The information that "aluminum is the third most abundant element" and that this contradicts aluminum as a causal agent has been successfully shared in an evidence map (media coordinations 27, 27a, 20, 19 and 20a). From an information sharing perspective, this sequence is sufficient to explain the fact that both the participants mentioned the abundance of aluminum (the successfully shared information) in rejecting aluminum as a disease factor. However, participants did another round trip for confirmation over 20 minutes later in the session (77a-8-8a). By exposing this dual round trip structure, the uptake analysis enabled us to hypothesize an interactional pattern in which information is first shared in one exchange, and then agreement on a joint interpretation of this information is accomplished in a second exchange. The analysis also helped us discover that participants accomplished the second confirmation round trip by moving to a different interactional medium, the threaded discussion.

We are often asked how long an analysis takes, and what tools we used. Time estimates that are predictive of future work are not yet possible, because the analyses reported in this paper took place concurrently with extensive discussions in which we developed the theoretical and practical basis for the framework. These discussions took place over many months with multiple revisions of the analyses. Visualizations of dependency graphs were constructed using standard tools such as Excel ${ }^{\mathrm{TM}}$, Visio ${ }^{\mathrm{TM}}$, and Omnigraffle ${ }^{\mathrm{TM}}$. Software tools tailored to this task will support more efficient analysis.

\section{Discussion}

The initial motivation for developing the dependency graph formalism was to support our analysis of collaborative knowledge construction through computer media (Suthers, 2006a). As this work progressed, we removed implicit assumptions about synchronicity and availability of contributions from the notation. We also realized that we could use the dependency graph as a boundary object between our different analysis methods. We used the dependency graph both to create aggregate statistics of interactions and their relationship to the media (Suthers, Vatrapu et al., 2007), and to examine the sequential structure of interaction (Suthers, Dwyer et al., 2007). The graph allowed us to trace asynchronous interaction between pairs of participants back from aspects of their essays that we wanted to explain. Our most recent analysis of the data (Suthers, Medina et al., 2007) bridged statistical and sequential approaches by algorithmically identifying instances of an interaction pattern we refer to as a "round trip" and then applying statistical tests on their frequency across experimental conditions.

There are multiple benefits to the dependency graph as a transcript notation. First, the notation is independent of the interaction medium and can be applied to face-to-face and online interactions as well as interactions that take place in multiple media. The use of generic media coordinations allows the inclusion of a 
Final draft of Suthers, D. D., Dwyer, N., Medina, R., \& Vatrapu, R. (2007). A framework for eclectic analysis of collaborative interaction. In C. Chinn, G. Erkens \& S. Puntambekar (Eds.), The Computer Supported Collaborative Learning (CSCL) Conference 2007 (pp. $694-703)$. New Brunswick: International Society of the Learning Sciences.

whole range of communicative actions, including perceptions and interactionally constructed representational elements. The concept of dependency extends the concepts of utterance and adjacency pair to online and asynchronous media and accounts for cases where media coordination is the result of multiple, previous media coordinations. Second, the notation can be used to address the tradeoff between statistical aggregation and sequential analysis described at the beginning of this paper. The dependency structure can be used to document and interpret the sequential structure of the interaction and can also be coded or searched to provide data for statistical analyses. Third, the dependency graph adapts to the density of the source data. High-fidelity data can be used to produce a dense graph that can be subject to detailed analysis. On the other hand, sparse data will produce a sparse graph but will still support limited analysis. Fourth, the graph data structure is open-ended-additional data can always be added, although this does imply that skepticism about the completeness of the graph should be maintained. Fifth, grounding in explicit media coordinations allows analysis of correlations between interaction patterns and the media affordances that shape them. Finally, the formalism of the graph structure supports building tools to manage its complexity and is amenable to algorithmic analysis and data mining techniques.

A delimitation of the framework is that, in focusing on observed interaction, it does not explicitly acknowledge the cultural or historical situatedness of the participants, or address identity and community, except where these constructs might be recorded in terms of prior interaction. Many theoretical and practical issues remain to be worked out. A pressing task is to extend the dependency graph formalism to better incorporate composite media coordinations and the possible ambiguity of dependencies. A complete explication of these two items is necessary to extend the potential algorithmic support provided by the dependency graph structure. The greatest practical need is to develop software tools to help construct and use the dependency graph. The need for improved analysis tools is a recurring theme (Sanderson \& Fisher, 1994), and the size and density of the potential data sets exacerbates this need. Elaborations on the visual representation should be explored, including embedding dependency graphs in a CORDTRA-style representation (Hmelo-Silver, 2003) to relate interaction to both media and episodes of activity. An important aspect of evaluating this framework will be to determine how well it scales to the types of interactions and media that are of most interest, including larger groups across longer time scales. Manual identification of media coordinations and dependencies is time-consuming at present, but with improved automation it might be possible to generate dependency graphs for larger online communities over the course of months or even years. Finally, the value of this framework in supporting multiple analytic traditions and producing "boundary objects" for CSCL research can only be realized in collaboration with other laboratories undertaking analysis of collaborative interaction.

\section{References}

Beck, I. L. (1997). Questioning the Author: An Approach for Enhancing Student Engagement with Text. Delaware: International Reading Association.

Bronckart, J. P. (1995). Theories of action, speech, natural language, and discourse. In J. V. Wertsch, P. D. Rio \& A. Alvarez (Eds.), Sociocultural Studies of Mind (pp. 75-91). New York: Cambridge University Press.

Chi, M., Bassok, M., Lewis, M., Reimann, P., \& Glaser, R. (1989). Self-Explanations: How Students Study and Use Examples in Learning to Solve Problems. Cognitive Science, 13, 145-182.

Clark, H. H., \& Brennan, S. E. (1991). Grounding in communication. In L. B. Resnick, J. M. Levine \& S. D. Teasley (Eds.), Perspectives on Socially Shared Cognition (pp. 127-149): American Psychological Association.

De Wever, B., Schellens, T., Valcke, M., \& Van Keer, H. (2006). Content analysis schemes to analyze transcripts of online asynchronous discussion groups: A review. Computers \& Education, 46(1), 6-28.

Doise, W., \& Mugny, G. (1984). The Social Development of the Intellect, International Series in Experimental Scoial Pscychology (Vol. 10): Pergamon Press.

Dwyer, N., \& Suthers, D. D. (2006). Consistent Practices in Artifact-Mediated Collaboration. International Journal of Computer-Supported Collaborative Learning, 1(4), 481-511.

Engestrom, Y. (2001). Expansive Learning at Work: toward an activity theoretical reconceptualization. Journal of Education and Work, 14(1).

Garcia, A. C., \& Jacobs, J. B. (1999). The Eyes of the Beholder: Understanding the Turn-Taking System in QuasiSynchronous Computer-Mediated Communication. Research on Language and Social Interaction, 32(4), 337-367.

Garfinkel, H. (1967). Studies in Ethnomethodology. Englewood Cliffs, New Jersey: Prentice-Hall.

Goodwin, C., \& Heritage, J. (1990). Conversation Analysis. Annual Review of Anthropology, 19, 283-307.

Herring, S. C. (1999). Interactional coherence in CMC. Journal of Computer Mediated Communication, 4(4). 
Final draft of Suthers, D. D., Dwyer, N., Medina, R., \& Vatrapu, R. (2007). A framework for eclectic analysis of collaborative interaction. In C. Chinn, G. Erkens \& S. Puntambekar (Eds.), The Computer Supported Collaborative Learning (CSCL) Conference 2007 (pp. $694-703$ ). New Brunswick: International Society of the Learning Sciences.

Hmelo-Silver, C. E. (2003). Analyzing collaborative knowledge construction: Multiple methods for integrated understanding. Computers \& Education, 41, 397-420.

Hutchins, E. (1995). Cognition in the Wild. Cambridge, Massachusets: The MIT Press.

Jordan, B., \& Henderson, A. (1995). Interaction analysis: Foundations and practice. Journal of the Learning Sciences, 4(1), 39-103.

Koschmann, T. (2002). Dewey's Contribution to the Foundations of CSCL Research. In G. Stahl (Ed.), Computer support for collaborative learning: Foundations for a CSCL community: Proceedings of CSCL 2002 (pp. 17-22). Boulder, CO: Lawrence Erlbaum Associates.

Koschmann, T., Zemel, A., \& Stahl, G. (2004). The video analyst's manifesto (or the implications of garfinkel's policies for studying practice within design-based research). In Y. Kafai, N. Sandoval, N. Enyedy, A. Nixon \& F. Herrera (Eds.), Proceedings of the Sixth International Conference of the Learning Sciences (pp. 278-385). Mahwah, NJ: Lawrence Erlbaum Associates.

Olson, G. M., Herbsleb, J. D., \& Rueter, H. H. (1994). Characterizing the sequential structure of interactive behaviors through statistical and grammatical techniques. Human-Computer Interaction, 9, 427-472.

Pea, R. (2006). Diver. Retrieved Nov. 26, 2006, from http://diver.stanford.edu

Resnick, L. B., Salmon, M., Zeitz, C. M., Wathen, S. H., \& Holowchak, M. (1993). Reasoning in conversation. Cognition and Instruction, 11(3\&4), 347-364.

Roschelle, J. (1992). Learning by Collaborating: Convergent Conceptual Change. The Journal of the Learning Sciences, 2(3), 235-276.

Roschelle, J., \& Teasley, S. D. (1995). The construction of shared knowledge in collaborative problem solving. In C. E. O'Malley (Ed.), Computer-Supported Collaborative Learning (pp. 69-197). Berlin: Springer-Verlag.

Rourke, L., Anderson, T., Garrison, D. R., \& Archer, W. (2000). Methodological Issues in the Content Analysis of Computer Conference Transcripts. International Journal of the Learning Sciences, 11.

Sacks, H., Schegloff, E. A., \& Jefferson, G. (1974). A Simplest Systematics for the Organization of Turn-Taking for Conversation. Language, 50(4), 696-735.

Sanderson, P., \& Fisher, C. (1994). Exploratory Sequential Data Analysis: Foundations. Human-Computer Interaction, 9, 251-317.

Schegloff, E. A., \& Sacks, H. (1973). Opening up closings. Semiotica, 8, 289-327.

Stahl, G. (2006). Group cognition: computer support for collaborative knowledge building. Cambridge, MA: MIT Press.

Stahl, G., Koschmann, T., \& Suthers, D. D. (2006). Computer-supported collaborative learning: An historical perspective. In R. K. Sawyer (Ed.), Cambridge handbook of the learning sciences (pp. 409-426). Cambridge, UK: Cambridge University Press.

Suthers, D. D. (2006a). A qualitative analysis of collaborative knowledge construction through shared representations. Research and Practice in Technology Enhanced Learning (RPTEL), 1(2), 1-28.

Suthers, D. D. (2006b). Technology affordances for intersubjective meaning-making: A research agenda for CSCL. International Journal of Computers Supported Collaborative Learning, 1(3), 315-337.

Suthers, D. D., Dwyer, N., Vatrapu, R., \& Medina, R. (2007). An abstract transcript notation for analyzing interactional construction of meaning in online learning. In Proceedings of the 40th Hawai $i$ International Conference on the System Sciences (HICSS-34), January 3-6, 2007, Waikoloa, Hawai i (CD-ROM): Institute of Electrical and Electronics Engineers, Inc. (IEEE).

Suthers, D. D., Medina, R., Vatrapu, R., \& Dwyer, N. (2007). Information Sharing is Incongruous with Collaborative Convergence: The Case for Interaction. In Computer Supported Collaborative Learning 2007 (this volume).

Suthers, D. D., Vatrapu, R., Medina, R., Joseph, S., \& Dwyer, N. (2007). Conceptual representations enhance knowledge construction in asynchronous collaboration. In Computer Supported Collaborative Learning 2007 (this volume).

Vygotsky, L. S. (1978). Mind in society. Cambridge, MA: Harvard University Press.

Wenger, E. (1998). Communities of Practice: Learning, Meaning and Identity. Cambridge: Cambridge University Press.

Wertsch, J. V. (1998). Mind as Action. New York: Oxford University Press.

Woods, D. (2006). Transana. Retrieved Nov. 26, 2006, from http://www.transana.org

\section{Acknowledgments}

This work was supported by the National Science Foundation under CAREER award 0093505. 